\title{
A review of the research progress in T-lymphocyte immunity and cervical cancer
}

\author{
Lina Zhang ${ }^{1,2 \#}$, Zhilei Mao ${ }^{1,2 \#}$, Yiqing Lai $^{1,2}$, Ting Wan ${ }^{1}$, Keliang Zhang ${ }^{1}$, Beibei Zhou ${ }^{1,2}$ \\ ${ }^{1}$ Department of Cervical Diseases Treatment, Changzhou Maternity and Child Health Care Hospital Affiliated to Nanjing Medical University, \\ Changzhou 213000, China; ${ }^{2}$ Centre for Diagnosis and Treatment of Cervical Diseases, Changzhou Maternity and Child Health Care Hospital, \\ Changzhou 213000, China \\ Contributions: (I) Conception and design: L Zhang; (II) Administrative support: K Zhang; (III) Provision of study materials or patients: Y Lai; (IV) \\ Collection and assembly of data: Z Mao; (V) Language and grammar check: T Wan; (VI) Manuscript writing: All authors; (VII) Final approval of \\ manuscript: All authors. \\ \#The authors contributed equally to this work. \\ Correspondence to: Dr. Beibei Zhou. Department of Cervical Diseases Treatment, Changzhou Maternity and Child Health Care Hospital Affiliated to \\ Nanjing Medical University, Changzhou 213000, China. Email: beibeizhou@njmu.edu.cn.
}

\begin{abstract}
Cervical cancer develops as a result of T-cell immune evasion by human papillomavirus (HPV). T-cell immunity requires the participation of many factors, such as antigen-presenting cells (APCs), cytokines, co-stimulatory molecules, etc. HPV vaccines are promising treatments to prevent HPV infection and cervical cancer. This article mainly provides a summary of the number and function changes of $\mathrm{T}$ cells during HPV infection and cervical cancer development. Studies on t-cell immunotherapy, which is expected to become a new treatment for cervical cancer after surgery, radiotherapy, and chemotherapy, are also reviewed in this article.
\end{abstract}

Keywords: T-cell; cervical cancer; human papillomavirus (HPV); immunotherapy

Submitted Dec 09, 2019. Accepted for publication Jan 08, 2020.

doi: $10.21037 /$ tcr.2020.01.33

View this article at: http://dx.doi.org/10.21037/tcr.2020.01.33

\section{Introduction}

Cervical cancer is one of the malignant tumours that seriously threaten the life and health of women. Nearly 500,000 women are diagnosed with cervical cancer each year (1), and nearly 288,000 of them are dying from the disease. It is widely acknowledged that high-risk human papillomavirus (HR-HPV) is a major risk factor for the development of cervical cancer, while persistent HR$\mathrm{HPV}$ infection is a high-risk factor for the recurrence of cervical intraepithelial neoplasia (CIN). Even though nearly $80 \%$ of HPV infections are transient (2), persistent infections are not necessary to develop cervical cancer. When HPV invades the human body, it triggers immune reactions, and cervical cancer results when HPV overcomes the immune system's responses (3). Immunity is responsible for the recognition and exclusion of foreign material and for maintaining the balance of the bodyfunctions which are closely related to the occurrence and development of tumours (4). Recent studies have shown that persistent infection of HPV has links with the body's immunity, particularly cervical T-cell immunity. Thus far, the majority of related studies have focused on tumour immunity therapy, but T-cell immunotherapy after surgical, radiological, or chemical therapy, is expected to become a new treatment method for cervical cancer. We require a more comprehensive understanding of the participating factors of T-cell immunity in order to clarify the changes in T-cell immunity in cervical tumour tissues and peripheral blood. It is only working from this level of comprehension that novel diagnosis and treatment methods for cervical cancer immunotherapy can be developed. This review provides a brief description of the relationship between T-cell immunity and cervical cancer. 


\section{Overview of $\mathbf{T}$ cells}

Thymus-dependent lymphocytes (abbreviated as T cells) are the main components of lymphocytes, comprising $65-75 \%$ of the total number of lymphocytes in peripheral blood and $>95 \%$ of lymphocytes in the thoracic duct. T cells act as "fighters" that resist diseases and tumours through varieties of biological effects, including the direct killing of target cells, regulating B cell antibody production, responding to specific antigens, and producing cytokines. T-cell immunity is called "cell immunity" because the T cells kill the targets directly instead of producing antibodies. $T$ cells can be divided into different subgroups according to different classification methods. Based on surface markers, they are divided by clusters of differentiation into $\mathrm{CD} 4^{+}$and $\mathrm{CD} 8^{+}$ $\mathrm{T}$ cells. Based on function, they are divided into cytotoxic $\mathrm{T}$ cells/lymphocytes (Tc, or CTLs), helper T (Th) cells, regulatory/suppressor $\mathrm{T}$ ( $\mathrm{T}$ reg) cells, and memory $\mathrm{T}$ cells.

\section{T-cell subsets and cervical cancer}

\section{Helper T cells}

Th cells can be classified into Th1, Th2, and Th17 subtypes. Th1 cells play crucial roles in cellular immunity, during which they secrete interferon-gamma (IFN- $\gamma$ ), interleukin (IL)-2, and tumour necrosis factor-beta (TNF- $\beta$ ) (5). These lymphokines further enhance and expand the immune response process and help stimulate other antitumour immunity processes. More specifically, IFN- $\gamma$ can inhibit tumour growth and enhance the anti-tumour activity of natural killer (NK) cells. Th2 plays a role in humoral immunity, which bolsters the production of antibodies from B cells, and Th2 may itself secrete IL-3, IL-4, IL5, IL-6, etc., of which IL-4 and IL-3 are known tumour growth factors which promote the rapid growth of tumour cells. IL-4 exerts an anti-tumour response by inhibiting the activation of NK cells and reducing tumour cell antigen expression. The Th1/Th2 ratio has proven to be a good indicator of the dynamic changes of the anti-tumour response, as the transfer of Th1 to Th2-type cells in the peripheral blood of cancer patients, especially those with cervical cancer, is responsible for the growth of malignant tumours and their evasion from the host's immunity response (5).

Th17 is the third independent Th cell subtype. In 2005, a study of the immunological pathogenesis of the central nervous system was first to identify Th17 (6). IL$17 \mathrm{~A}$ is secreted by Th17 and exerts a strong immune effect in the early stages of many inflammatory diseases. As a consequence, the Th17/Th1 ratio was used to assess the changes in immunity (7). Another cytokine secreted by Th17, IL-23, can block certain extracellular pathogens and has a crucial role in inflammation response and autoimmune diseases (2). A study that focused on the transcriptional regulation of Th17 expression evidenced that autoimmune diseases were pathogenically driven by IL-17/IFN- $\gamma$ double-producing cells (8).

In recent years, the relationship between Th17 cells and cervical cancer has become an area of intense research focus. Studies have revealed that Th17 cells are elevated in patients with cervical cancer and in those with CIN. The higher levels of Th22, Th17, and Th1 cells in the circulation system may responsible for the pathogenesis of cervical cancer (9). Clinical stage, lymph node metastasis, and invasion were proven to be associated with the increased Th17 cell level in cervical cancer patients, whereas increased $\mathrm{T}$ reg cell frequency was associated with tumour differentiation. Furthermore, the Th17/T reg ratio was found to be significantly higher in patients with uterine cervical cancer with lymph node metastasis or invasion than in patients without cancer (10). A similar study showed that the Th17/T reg balance was broken, and the related cytokines were significantly higher in the blood of Uygur patients with cervical cancer or CIN (11). The imbalance of $\mathrm{T}$ cells that expressed Th17/fork head box protein 3 (FOXP3) is responsible for the development of uterine cervical cancer by fostering angiogenesis (12). Though the roles of Th17 cells in promoting autoimmune disorders and inflammation responses have been extensively demonstrated, whether and how Th17 cells affect tumour immunity response are still controversial. On one hand, Th17 cells can promote tumour development by inducing angiogenesis and suppressing immune functions. On the other hand, Th17 cells can activate anti-tumour responses by recruiting immune cells to the tumours and activating $\mathrm{CD}^{+} \mathrm{T}$ cells, or by activating the phenotype converting Th1 and producing IFN- $\gamma$. This characteristic of Th17 cells might be used for developing it as a target in future therapeutic strategies (13).

\section{Regulatory/suppressor T cells}

$\mathrm{T}$ reg cells, which are a group of cells with special phenotypes and functions, can be divided into native $T$ reg cells and induced $\mathrm{T}$ reg cells depending on their source. Native $T$ reg cells mainly maintain immune tolerance and 
immune homeostasis (14). Induced T reg cells can inhibit the anti-tumour response and consequently promote the development of tumours. For instance, $\mathrm{CD}^{+}{ }^{+} \mathrm{T}$ reg cells are involved in the anti-tumour response when it is an important component of specific immunity. Since being discovered in previous studies, the transcription factor FOXP3 has become the most commonly and widely used marker for $\mathrm{T}$ reg cells, whose expression is closely related with the survival of invasive carcinoma patients (15).

A related study of cervical cancer found that large numbers of CD4 and CD25 cells were recruited by $\mathrm{T}$ reg cells in the blood of patients with high-grade squamous intraepithelial lesions (16). The $\mathrm{T}$ reg cell numbers in the blood of the cervical cancer and CIN patients were significantly higher than that in the control patients (11). Similar studies reported that $\mathrm{T}$ reg cell levels in cervical cancer patients were correlated with the stages of the disease (staged by the Federation International of Gynecology and Obstetrics staging system) and the degree of tumour differentiation, which indicates that $\mathrm{T}$ reg cells are closely related to HPV infection (17). A large number of studies have confirmed that $\mathrm{T}$ reg cells are associated with cervical immune regulation. As a marker of T reg cells, FOXP3 increment is accompanied by an increased number of cervical lesions and lower tumour cell differentiation (18). In cervical cancer, $\mathrm{T}$ reg cells dominate the microenvironment of tumour-positive lymph nodes in high frequencies, thereby increasing the deterioration risk of patients (19). $\mathrm{FOXP}^{+}$Treg cells accumulate in the stroma of CIN3, micro- infiltration, and invasive cancers, while FOXP $3^{+}$Treg cells gather in the lymph nodes of metastatic invasive cancer, thus possibly creating a network for immune escape (20).

Th17 and T reg cells are unlikely allies, and have broad prospects for the treatment of autoimmune diseases and cancers in humans. $\mathrm{T}$ reg cells were shown to promote Th17 cell differentiation in vitro and in vivo, and enhance the function of Th17 cells, which included a protective effect in the host's defence. In contrast, they exerted detrimental effects in inflammation and in the support of tumour growth. An in vivo study suggested that Th17 cells are the most effective Th subtype in stimulating and supporting the phenotypic stability and expansion of $\mathrm{T}$ reg cells. These observations suggest that two kinds of Th cells may reciprocally stimulate each other, the effects of which should be considered when future Th17 and T reg celltargeted therapies are conducted (21).

\section{Changes in $\mathrm{CD} 4$ and $\mathrm{CD} 8$}

CD4 and CD8 are both glycoproteins and differentiated by their morphology, which is expressed on the surface of $\mathrm{T}$ cells as cell receptors. CD4 is expressed mainly on Th and T reg cells, whereas CD8 is expressed mainly on CTLs. Most studies have provided evidence that $\mathrm{T}$ lymphocyte subsets are abnormal and have imbalanced proportions in patients with tumours of various types. One study showed that the ratio of $\mathrm{CD}^{+}$to $\mathrm{CD}^{+}$subtypes in cervical cancer tissue was significantly lower than that in the peripheral blood, and this alteration was more obvious in higher stages of the disease (22). The decreased proportions of tumour-infiltrated $\mathrm{CD}^{+}{ }^{+} \mathrm{T}$ cells, as well as the reversed $\mathrm{CD} 4^{+} / \mathrm{CD} 8^{+}$ratio, are closely related with rapid tumour growth and lymph node metastasis of cervical cancer patients. Regional immune escape is of great importance to cancer progression (23). In regressing $\mathrm{CIN} 1, \mathrm{CD} 4^{+}$cells predominate within both the stroma and epithelium, presenting the highest $\mathrm{CD}^{+} /$ $\mathrm{CD}^{+}$ratio compared with the progressing CIN1, CIN3, and invasive carcinoma (24). The $\mathrm{CD}^{+} / \mathrm{CD}^{+}$ratio is also associated with the 5 -year survival rate of cervical cancer, patients with a lower $\mathrm{CD}^{+} \mathrm{T}$ reg cell level with the upregulated $\mathrm{CD}^{+} / \mathrm{CD}^{+}$ratio usually obtaining better clinical outcomes (25). Another study showed that the expression of $\mathrm{CD}^{+}$and $\mathrm{CD}^{+}$was inhibited or even deleted in CIN and cervical cancer (26). It was also found that in the early stage of cervical cancer, $\mathrm{CD} 8^{+}$cells and $\mathrm{CD} 8^{+} / \mathrm{CD} 4^{+}$ratio were significantly elevated in patients without lymph nodes metastasis. When the $\mathrm{CD} 8^{+} / \mathrm{CD}^{+}$ratio decreased below 2 , both the total survival rate and disease-free survival rate decreased, a finding which was also reported in other research studies $(11,27)$.

Unlike CD4 and CD8 antigens, CD3 is expressed on all subsets of $\mathrm{T}$ cells. The density of peritumoral $\mathrm{CD}^{+}$cells was found to have the strongest ability in relapse prediction, and the hazard ratio reduced to 0.27 when the $\mathrm{CD}^{+}$cell density increased from 795 to 2,043 cells $/ \mathrm{mm}^{2}$ (28).

\section{T-cell immunity and cervical cancer}

HR-HPV, a major risk factor for cervical cancer development, can produce two kinds of oncoproteins (viz. E6 and E7), which affect cell cycle and inhibit cell apoptosis. E6 and E7 are closely related to the genesis and development of tumours. When these two oncoproteins are recognized, and their expression is then inhibited by T-cellspecific immunity, this is known as an anti-tumour immune 
response. At present, a large number of studies are being performed to investigate $\mathrm{HPV}$-specific immunity and the immunosuppression of cervical cancer.

\section{Antigen-presenting cells (APCs)}

APCs exert critical roles for the antigen transportation from the periphery to locally organized lymphoid tissue (29). Dendritic cells (DCs) are a group of APCs that possess unique abilities to activate primary immune responses. DCs capture antigen and transfer the antigen information to activate adaptive immune response. They are not only crucial for primary immune responses, but also important for immune tolerance and $\mathrm{T}$ cell-mediated immune regulation (30). DCs can be precisely and effectively delivered in vivo by optimally adjusting the net charge after intravenous administration of RNA-lipoplexes. This thus mediates the efficient uptake and expression of antigens including major histocompatibility complex (MHC), costimulatory molecules, and adhesion molecules, which are required for T-cell activation by the DC populations (31). As the most professional APCs, they induce strong effector and memory T-cell response and mediate IFNdependent rejection in progressive melanoma tumours (31). Furthermore, DCs produce IL-12, a cytokine crucial for anti-tumour responses, after strong activation (32). CD $83^{+}$DCs can be found in cancer biopsies, and phenotypically mature CD83 $3^{+}$DCs in cancer cells express matrix metalloproteinase 9 (MMP-9), a kind of protein known to have vasculogenic and tumor-genesis-promoting properties (33). Studies reported that a massive amount of $\mathrm{MHC}$ class $\mathrm{II}^{+} \mathrm{CD} 83^{+}$DCs were observed in the intratumoral region of $\mathrm{HPV}$-induced head and neck cancer, where the maturation of DCs (assessed by the CD83/ DC-SIGN ratio) was significantly lower than that in the peritumoral stroma $(\mathrm{P}<0.05)$, indicating a distinct immune regulatory mechanism between the intra- and peritumoral sites (34). DC-derived exosomes (Dexo) were demonstrated to join ex vivo and in vivo anti-tumour immune responses. Dexo loading the $\mathrm{E} 7_{49-57}$ peptide can efficiently activate $\mathrm{CD}^{+} \mathrm{T}$ cells against tissue culture-1 (TC-1) tumour cells, via promoting their proliferation and excretion of IFN- $\gamma$ ex vivo (35). NK cells were also shown to enhance DC maturation and secretion of CD86, human leukocyte antigen-DR isotype (HLA-DR), and IL-12p70 in the presence of HPV virus (36). The DCs further activated NK cells by up-regulating cell surface markers (CD69 and HLA-DR), secreting IFN- $\gamma$ and activating cytotoxicity toward an HPV-positive cell line. Another study showed that monocyte-derived DCs that pulsed with recombinant Mycobacterium tuberculosis heat shock protein 70-formyl peptide receptor 1 (MtHSP70-FPR1) expressed high levels of HLA-DR, CCR7, CD80, CD83, and CD86, and secreted more TNF-a, IL-12p70, and IL- $1 \beta$ to induce stronger CTL activation and proliferation, thus resulting an effective killing effect of cervical cancer cells (37).

\section{Cytokines}

Cytokines are soluble low molecular weight proteins which are produced by many immune-related cells under the stimulation of antigens and exhibit immune regulatory functions, including ILs, IFNs, TNFs, etc. Research indicates that, with the progression of CIN lesions in the uterine cervical increased, the expression of IL- $1 \beta$ and IL-8 is enhanced, meaning that the Th2 inflammatory response is strong. Meanwhile, a decrease in the macrophage inflammatory protein-1beta (MIP-1 $\beta$ ) level may be helpful for the immune escape of HPV (38). The levels of IL10, IL-17, IL-23, and transforming growth factor-beta (TGF- $\beta$ ) were more highly expressed in the peripheral blood of patients infected with high-risk cervical HPV than in those infected with low-risk cervical HPV $(\mathrm{P}<0.05)(39)$. The logistic regression analysis indicated that, after adjusting for other variables (age, cervical cancer history, and smoking history), the single nucleotide polymorphism of three Th2 cytokines (IL-4, IL-6, and IL-10) and one Th3 cytokine (TGF- $\beta 1$ ) were found to have a significant association with cervical cancer (40). Tumour necrosis factor receptor 2 (TNFR2) $^{+} \mathrm{T}$ reg cells and relevant cytokines have been confirmed to be associated with cervical cancer development. Both the peripheral and tumour-infiltrated TNFR2 ${ }^{+}$Treg cells were found to be significantly higher in patients with CIN or cervical cancer, and the percentage of peripheral TNFR2 ${ }^{+}$Treg cells was negatively correlated with the clinical stages of cervical cancer (41). In HPVpositive head and neck squamous cell carcinoma, the defection of TNF receptor-associated factor 3 (TRAF3) activated an alternative nuclear factor-kappa B signalling, which suppressed the expression of anti-viral cytokine and promoted cancer cell survival and resistance (42). A higher serum TNF- $\alpha$ is level was also shown to contribute to a lower clearance of oral infected HPV in men than in women (43). HPV possibly interferes with the trafficking of leukocyte by synthesizing and releasing specific proinflammatory cytokines and chemokines to change the 
tumour micro-environment, which may be helpful for tumour development (44).

\section{Co-stimulatory molecules}

Co-stimulatory signals are important for T-cell activation. Although $\mathrm{CD}^{+} \mathrm{T}$ cells induced by APCs lacking costimulatory molecules were found to retain their cytotoxic activity, the lack of cloning and proliferation ability was observed and their abilities on adaptive immune response and IL-2 secretion were inhibited (45). Previous studies showed that HPV could escape from the host immunity system. The HPV-mediated suppression of co-stimulatory signals by Langerhans cells (LCs) may be responsible for persistent HPV infection and immune escape (46). When the proper stimulus was given to women with HPV persistent infection history, then the LCs presented HPV antigens and induced an adaptive T-cell immune response. For instance, stabilized polyinosinic: polycytidylic acid [s-Poly(I:C)] compounds are indicated to be a promising immune-modulator for the clearance of persistent HPV infection (47).

The B7 family consists of the following important costimulatory molecules: B7-H1, B7-H2 (ICOSL), B7-1 (CD80), B7-2 (CD86), B7-H3 (CD276), programmed death-ligand 1 (PD-L1), B7-H4 (B7X), and B7-DC (PDL2). The interactions between B7 molecules and CD28 family receptors are crucial for adaptive cellular immunity regulation. In cancer, the aberrant expression of coinhibitory B7 was demonstrated to reduce anti-tumour response and was attributed to cancer immune escape. In addition, cytotoxic T-lymphocyte-associated protein-4 (CTLA-4) could reduce the level of B7 ligands and inhibit T-cell response, mainly via inhibiting IL-2 and blocking cell cycle progression (48).

Co-stimulatory receptors on the T cell surface (e.g., CD28) could bind with the co-stimulatory molecules on the APC surface, including CD80 (B7-1) and CD86 (B7-2). The combination could then trigger the survival and proliferation of activated $\mathrm{T}$ cells (signal 2), which is important for immune tolerance and T-cell responsiveness regulation (49). B7-H3 co-stimulates the proliferation of both $\mathrm{CD}^{+}$and $\mathrm{CD} 8^{+} \mathrm{T}$ cells, which enhances the induction of CTLs and stimulates IFN- $\gamma$ production via T-cell receptor signalling (50). In addition, B7 molecules may promote the proliferation, invasion and migration of cancer cells (51). The expression level of $\mathrm{B} 7-\mathrm{H} 3$ is significantly higher in cervical cancer tissues than in normal tissues, indicating a worse prognosis for patients (52). The HPV E6 and E7 oncoproteins have strong antigenicity. The introduction of B7 molecules into cervical cancer cells could enhance the immunogenicity of the cells via synergistic stimulation and the down-regulation of immunosuppression, thus inhibiting their proliferation and invasion. They may therefore be a potential target for cervical cancer therapy.

\section{Cervical cancer immunotherapy}

At present, cervical cancer can be prevented by HPV vaccine injection and regular cervical cancer screening, but protective vaccines cannot eliminate an existing HPV infection and cannot prevent the development of cervical precancerous lesions or cervical cancer. Therefore, it is urgent to find effective counter measures for existing HPV infections and related diseases.

\section{Adoptive T-cell therapy}

The adoptive transfer of tumour-specific $\mathrm{T}$ cells, which are obtained from tumour-infiltrating lymphocytes or peripheral blood, is a promising immunotherapeutic approach for cancer. In a clinical trial, 9 patients, who were diagnosed with metastatic cervical cancer, were given a signal infusion of tumour-infiltrated $\mathrm{T}$ cells that were HPV E6 and E7 reactive after finishing platinumbased chemotherapy or chemoradiotherapy; the tumour completely regressed in 2 patients (53). After treatment, 2 complete responses were ongoing at 22 and 15 months, respectively, and 1 partial response was received at 3 months into the trial (53).

The tumour-draining lymph nodes of patients with HPVinduced cervical cancer can be used as a source for adoptive cell transfer. One study demonstrated that the stimulation of lymph node mononuclear cells from the tumour-draining lymph nodes resulted in a 36-fold increment of polyclonal HPV-specific $\mathrm{T}$ cells under a clinical grade condition for adoptive immunotherapy in patients with cervical cancer (54).

\section{Inbibitors of immune checkpoints}

In normal conditions, immune checkpoints are crucial for the maintenance of self-tolerance, which prevents autoimmunity and normal cells from being attacked by the immune system. Unfortunately, cancer cells can utilize this function to escape from immunization surveillance. Drugs 
that block the immune checkpoints (viz. the programmed death protein-1 (PD-1)/PD-L1 and CTLA-4 pathway) have shown promising results for solid and hematological malignancies by significantly improving the survival of newly diagnosed and heavily pre-treated patients (55).

In 2014, the US Food and Drug Administration (FDA) approved a humanized antibody against PD-1 (Pembrolizumab, Merck \& Co. Inc., White house Station, NJ, USA) and a human anti-PD-1 antibody (Nivolumab, Bristol-Myers Squibb, New York, NY, USA) to treat advanced melanoma (56,57). Recently, a complex method consisting of anti-PD-1 antibody (CT-011), low-dose cyclophosphamide depletion of $\mathrm{T}$ reg cell, and the HPV 16 E7 peptide vaccine, was shown to activate antigen-specific immune responses and induce the complete regression of tumours to prolong the survival time of a significant percentage of treated animals (58). Another study showed that tumours may form an "immune-privileged" region for adaptive immune resistance via the PD-1/PDL-1 pathway, which may reasonably explain the therapeutic hypo-responsiveness of this pathway in HPV-positive oropharyngeal squamous cell carcinoma patients (59).

New methods are emerging in cancer immunotherapy beyond CTLA-4 and PD-1. PD-L1 and lymphocyteactivation gene-3 (LAG-3) inhibitors have made progress in clinical trials. B7 family members and the TNFR superfamily members have also shown promise or variable success in preclinical models and clinical studies, respectively (60).

\section{Therapeutic HPV vaccines}

Recently, various therapeutic HPV vaccines are being studied with the aim to activate an HPV-associated T-cell immune response and eliminate HPV-related diseases and cancer. The HPV E6 and E7 tumour antigens are expressed only on cervical cancer cells surface, but not in normal tissues (61), which make them ideal targets for therapeutic vaccines that do not damage normal tissue.

\section{Live vaccines}

Live vaccines can activate strong cellular and humoral immune response (62), and can be classified into bacterial- and viral-based vectors, for which Lactobacillus casei and Listeria monocytogenes have been widely used to develop vaccines for HPV infection therapy. In a study which was the first to report a correlation between cellmediated immune response specific to E7 in the cervix after immunotherapy for human mucosal neoplasia, oral administration of a Lactobacillus-based vaccine expressing E7 activated a specific mucosal immunity to E7 in uterine cervical lesions. About $70 \%$ of the patients experienced a pathological downgrade from CIN3 to CIN2 after taking the optimized dose (63).

The immunotherapy ADXS11-001 is a live attenuated Listeria monocytogenes cancer vaccine that secretes a fusion product of the HPV16E7 antigen and listeriolysin $\mathrm{O}$ protein-targeting HPV-transformed cells. A phase I clinical trial showed that patients with persistent/recurrent metastatic cervical cancer who received a high ADXS11001 tolerated it well, and the most common treatmentrelated adverse events (TRAEs) that occurred in $\geq 3$ patients were chills, vomiting, hypotension, tachycardia, fever, and nausea (64). Only one grade 3 TRAE (hypotension) and no grade 4 or grade 5 TRAEs were reported in the study (64). In a phase II clinical trial, $69 \%$ of patients $(18 / 26)$ who received all three per-protocol doses of ADXS11-001 had a median survival duration of $>1$ year [12.1 (95\% CI: 6.8-NR) months], and the 12 -month overall survival rate was $55.6 \%$. The Stage 2 trail is currently recruiting patients and has been amended to allow for continuous cycles of therapy (65).

Viral vectors enter host cells with high efficiency and can activate the T-cell responses. For safety reasons, viral vectors are limited to use in patients who are immunocompromised. In a first stage clinical trial, a recombinant vaccinia virus was administrated to 8 patients with advanced stage of cervical cancer (66). The result showed that this kind of vaccination generated only a mild and tolerable response in patients with single-dose inoculation. Another clinical study that was performed in 29 patients with stage IB or IIA cancer with 2 -time injection of TA-HPV vaccine showed that $>28 \%$ of the patients exhibited specific serological responses, but the CTL response was transient (67).

\section{Peptide vaccines}

Compared to live virus vaccines, peptide vaccines are easier to produce, more stable, and safer. However, single epitope-based peptide vaccines tend to generate a narrow and weak immune response. Researchers proposed using overlapping peptides or mixed overlapping long peptides as vaccines to remedy this weakness (68). One study showed that the HPV16 E-and-E7-overlapping long peptide-based vaccine was well-tolerated and capable of inducing a broad IFN- $\gamma$-associated T-cell response even in patients with advanced cervical cancer (69). Other clinical research has demonstrated that a mixed overlapping long peptide vaccine 
could generate a complete or partial response in $47 \%$ and $80 \%$ of the participants, respectively (70). A recent clinical trial study showed that the combination of an overlapping long peptide vaccine with immune-check point inhibitor was more efficient than the use of the check point inhibitor alone (71).

\section{Protein vaccines}

TA-CIN, a 725-amino-acid-based protein vaccine, has been shown to generate HPV16-specific antibodies and CTL responses in TC-1 tumour-bearing mice. In a phase I clinical trial, though no adverse events were observed, the HPV16 E6- and E7- specific responses were low (72). In another clinical study, a group of CIN 3 patients received a loop electrosurgical excision procedure or cone biopsy, with $78 \%$ of them presenting complete or partial responses, and two patients $(3.5 \%)$ progressing to microinvasive cancer after they were subcutaneously injected with SGN-00101. No other drug-related adverse effects were reported (73).

\section{Recombinant overlapping peptide vaccines}

Mixed synthetic overlapping peptides or long peptides vaccines have many advantages, but challenges in manufacture quality control and the drug registration have arisen. Although protein-based vaccines have less of the above-mentioned disadvantages, they only stimulate CD4 T and $\mathrm{B}$ cell responses and not the $\mathrm{CD} 8 \mathrm{~T}$ cell response.

Considering the disadvantages of synthetic overlapping peptides and protein-based vaccines, the recombinant overlapping peptide was proposed as a new category of vaccine. To put briefly, the overlapping peptide sequences of a target protein are linked to an intracellular protease. The linked sequences are then expressed as a recombinant protein (also called recombinant overlapping peptides, ROP). ROPs have been tested as vaccines successfully in proof-of-concept in vitro and in vivo studies (74). The ROP vaccine can be produced as one entity to avoid the multiple disadvantages in quality control and registration. Moreover, ROP vaccines can be cleaved into overlapping peptides to stimulate both CD4 and CD8 $\mathrm{T}$ cell responses once they are ingested by APCs (74).

\section{DNA vaccines}

The DNA vaccine has emerged as a promising immunotherapeutic agent against cancers by virtue of its stability, simplicity and safety. A number of clinical trials have demonstrated that the DNA vaccines is well-tolerated by patients and does not trigger significant adverse effects (75).
More recently, sequential pNGVLa-Sig/E7(detox)HSP70, with a recombinant vaccine virus encoding an immunomodulatory E6/E7 fusion protein, was tested in 12 patients with CIN3 for the phase I trial, resulting in 5 of the subjects showing complete tumour regression (76). A phase I-II test of the VGX-3100 vaccine (a mixture of two plasmids encoding HPV16/18 E6/E7 antigens) showed that $49.5 \%(53 / 107)$ of the patients treated with CIN2/3 who were administered by electroporation showed complete tumour regression. VGX-3100, by inducing both T-cell and B-cell responses to E6 and E7, was proven to be the first effective therapeutic vaccine against HPV 16- and HPV18associated CIN2/3 (77).

Despite these advantages, DNA vaccines for cancers still entail a few disadvantages, including the relatively low immunogenicity, which have impeded the expected success of clinical application. Naked DNA is not easily spread between cells in vivo, and APCs do readily take up and present antigens to start effective immune responses. Thus, effective strategies need to be explored to enhance the potency of DNA vaccines.

\section{DC-based vaccines}

DCs possess strong abilities in activating and controlling the T-cell response, which makes them ideal candidates for immunotherapy strategies. A previous study showed that DCs stimulated with E6/E7 RNA could activate antigen-specific CTL to recognise and lyse DCs that were transfected with E6 or E7 RNA and cervical carcinoma cells expressing E6/E7 proteins. The efficiency was similar to that which the researchers achieved in DCs that were activated with E6 and E7 peptides. Moreover, DCs cotransfected with both E6/E7 RNA showed more efficiency in stimulating CTLs to lyse human cervical cancer cells (78). A modified HPV16 E7-pulsed DC vaccine enhanced by an adenovirus-mediated suppressor of cytokine signalling 1 (SOCS1) reduced the transformation activity and improved its antigenicity, thus receiving a better immunotherapeutic effect in allografted tumour mouse models (79).

Collectively, Dexo loaded with the E7 49-57 peptide could effectively induce $\mathrm{CD} 8^{+} \mathrm{T}$ cell proliferation and IFN- $\gamma$ excretion to exert cytotoxic activity toward TC-1 tumour cells ex vivo. Moreover, the Dexo vaccine promoted the re-stimulation responses of $\mathrm{E} 7$ antigen vaccinated mouse splenocytes in vitro. Notably, Poly (I:C) dramatically increased the potent anti-tumour ability of antigen-pulsed Dexo in cervical cancer amelioration (35). Although various 
cellular immunotherapies have made substantial progress in the treatment of HPV-related diseases, further research needs to be performed and large-scale clinical data need to be collected to confirm their efficacy.

\section{Conclusions}

Cell immunity is closely related to the development of cervical cancer. APCs, co-stimulatory molecules, cytokines, and HPV subtypes are also involved in the immune response of cervical cancer. $\mathrm{CD} 8^{+} \mathrm{T}$ cells and the ratio of $\mathrm{CD} 4^{+} / \mathrm{CD} 8^{+}$may influence the immune function of patients. A review of the relevant studies was provided above with the of aim creating a platform for future research studies to derive effective treatments from the various specific T-cell immune responses, and to use the effective immune response to control tumour development and improve therapy.

\section{Acknowledgments}

Funding: This study was supported by the Jiangsu Provincial Medical Youth Talent (QNRC2016303), the Medical Scientific Research Projects of the Health and Family Planning Commission in Jiangsu province (H2018018), and the Funding Project of Science and Technology Development in Nanjing Medical University (2017NJMUZD045).

\section{Footnote}

Conflicts of Interest: All authors have completed the ICMJE uniform disclosure form (available at http://dx.doi. org/10.21037/tcr.2020.01.33). The authors have no conflicts of interest to declare.

Ethical Statement: The authors are accountable for all aspects of the work in ensuring that questions related to the accuracy or integrity of any part of the work are appropriately investigated and resolved.

Open Access Statement: This is an Open Access article distributed in accordance with the Creative Commons Attribution-NonCommercial-NoDerivs 4.0 International License (CC BY-NC-ND 4.0), which permits the noncommercial replication and distribution of the article with the strict proviso that no changes or edits are made and the original work is properly cited (including links to both the formal publication through the relevant DOI and the license). See: https://creativecommons.org/licenses/by-nc-nd/4.0/.

\section{References}

1. Li H, Yang Y, Zhou H, et al. Clinicopathologic characteristics and survival analysis in stage IVB cervical cancer with hematogenous metastasis. Transl Cancer Res 2019;8:1217-23.

2. Zambrano-Zaragoza JF, Romo-Martinez EJ, Duran-Avelar Mde J, et al. Th17 cells in autoimmune and infectious diseases. Int J Inflam 2014;2014:651503.

3. Marks MA, Viscidi RP, Chang K, et al. Differences in the concentration and correlation of cervical immune markers among HPV positive and negative perimenopausal women. Cytokine 2011;56:798-803.

4. Ouyang P, Wu K, Su L, et al. Inhibition of human cervical cancer cell invasion by IL-37 involving runt related transcription factor 2 suppression. Ann Transl Med 2019;7:568.

5. Del Prete G. Human Th1 and Th2 lymphocytes: their role in the pathophysiology of atopy. Allergy 1992;47:450-5.

6. Xin J, Wainwright DA, Serpe CJ, et al. Phenotype of CD4+ $T$ cell subsets that develop following mouse facial nerve axotomy. Brain Behav Immun 2008;22:528-37.

7. Rostami A, Ciric B. Role of Th17 cells in the pathogenesis of CNS inflammatory demyelination. J Neurol Sci 2013;333:76-87.

8. Stadhouders R, Lubberts E, Hendriks RW. A cellular and molecular view of $\mathrm{T}$ helper 17 cell plasticity in autoimmunity. J Autoimmun 2018;87:1-15.

9. Zhang W, Tian X, Mumtahana F, et al. The existence of Th22, pure Th17 and Th1 cells in CIN and Cervical Cancer along with their frequency variation in different stages of cervical cancer. BMC Cancer 2015;15:717.

10. Zhang Y, Ma D, Zhang Y, et al. The imbalance of Th17/ Treg in patients with uterine cervical cancer. Clin Chim Acta 2011;412:894-900.

11. Chen Z, Ding J, Pang N, et al. The Th17/Treg balance and the expression of related cytokines in Uygur cervical cancer patients. Diagn Pathol 2013;8:61.

12. Hou F, Li Z, Ma D, et al. Distribution of Th17 cells and Foxp3-expressing $T$ cells in tumor-infiltrating lymphocytes in patients with uterine cervical cancer. Clin Chim Acta 2012;413:1848-54.

13. Guery L, Hugues S. Th17 Cell Plasticity and Functions in Cancer Immunity. Biomed Res Int 2015;2015:314620. 
14. Wei M, Shen D, Mulmi Shrestha S, et al. The Progress of T Cell Immunity Related to Prognosis in Gastric Cancer. Biomed Res Int 2018;2018:3201940.

15. Zheng S, Shen Y, Song Y, et al. How to detour Treg cells in T cell-based antitumor immune therapy. Onco Targets Ther 2013;6:1243-7.

16. Bonin CM, Padovani CT, Ferreira AMT, et al. Predominant overexpression of CD25/FOXP3, IFN- $\gamma$, and suppressive cytokines in high-grade lesion samples infected with human papillomavirus. Jornal Brasileiro de Patologia e Medicina Laboratorial 2017;53:53-60.

17. Zhang J, Liu B, Zhang Y, et al. Changes of CD4+ CD25+ CD127low regulatory $T$ cells in peripheral blood samples collected from the patients with cervical cancer and its clinical significance. Chinese Journal of Microbiology and Immunology 2015;35:753-8.

18. Pang X, Zhang Y, Wei H, et al. Expression and effects of high-mobility group box 1 in cervical cancer. Int J Mol Sci 2014;15:8699-712.

19. Heeren AM, de Boer E, Bleeker MC, et al. Nodal metastasis in cervical cancer occurs in clearly delineated fields of immune suppression in the pelvic lymph catchment area. Oncotarget 2015;6:32484-93.

20. Nakamura T, Shima T, Saeki A, et al. Expression of indoleamine 2, 3-dioxygenase and the recruitment of Foxp3-expressing regulatory $\mathrm{T}$ cells in the development and progression of uterine cervical cancer. Cancer science 2007;98:874-81.

21. Chen X, Oppenheim JJ. Th17 cells and Tregs: unlikely allies. J Leukoc Biol 2014;95:723-31.

22. Das D, Sarkar B, Mukhopadhyay S, et al. An Altered Ratio of CD4+ And CD8+ T Lymphocytes in Cervical Cancer Tissues and Peripheral Blood - A Prognostic Clue? Asian Pac J Cancer Prev 2018;19:471-8.

23. Sheu BC, Hsu SM, Ho HN, et al. Reversed CD4/CD8 ratios of tumor-infiltrating lymphocytes are correlated with the progression of human cervical carcinoma. Cancer 1999;86:1537-43.

24. Monnier-Benoit S, Mauny F, Riethmuller D, et al. Immunohistochemical analysis of CD4+ and CD8+ T-cell subsets in high risk human papillomavirus-associated premalignant and malignant lesions of the uterine cervix. Gynecol Oncol 2006;102:22-31.

25. Shah $W$, Yan $X$, Jing L, et al. A reversed CD4/CD8 ratio of tumor-infiltrating lymphocytes and a high percentage of CD4+ FOXP3 + regulatory $\mathrm{T}$ cells are significantly associated with clinical outcome in squamous cell carcinoma of the cervix. Cell Mol Immunol 2011;8:59.
26. Fan JT, Liao Y, Si XH, et al. Expression of HLA-I, CD8, and CD4 and Their Clinical Significance in Cervical Cancer. World J Oncol 2011;2:10-5.

27. Kurmyshkina OV, Kovchur PI, Schegoleva LV, et al. Tand NK-cell populations with regulatory phenotype and markers of apoptosis in circulating lymphocytes of patients with CIN3 or microcarcinoma of the cervix: evidence for potential mechanisms of immune suppression. Infect Agent Cancer 2017;12:56.

28. Nedergaard BS, Ladekarl M, Thomsen HF, et al. Low density of CD3+, CD4+ and CD8+ cells is associated with increased risk of relapse in squamous cell cervical cancer. Br J Cancer 2007;97:1135-8.

29. Zinkernagel RM, Ehl S, Aichele P, et al. Antigen localisation regulates immune responses in a dose- and time-dependent fashion: a geographical view of immune reactivity. Immunol Rev 1997;156:199-209.

30. Banchereau J, Briere F, Caux C, et al. Immunobiology of dendritic cells. Annu Rev Immunol 2000;18:767-811.

31. Kranz LM, Diken M, Haas H, et al. Systemic RNA delivery to dendritic cells exploits antiviral defence for cancer immunotherapy. Nature 2016;534:396-401.

32. Schmidt SV, Seibert S, Walch-Ruckheim B, et al. RIPK3 expression in cervical cancer cells is required for PolyIC-induced necroptosis, IL-1alpha release, and efficient paracrine dendritic cell activation. Oncotarget 2015;6:8635-47.

33. Pahne-Zeppenfeld J, Schroer N, Walch-Ruckheim B, et al. Cervical cancer cell-derived interleukin-6 impairs CCR7dependent migration of MMP-9-expressing dendritic cells. Int J Cancer 2014;134:2061-73.

34. Tsujikawa T, Borkar RN, Azimi V, et al. Multiplex immunohistochemistry for immune profiling of HPVassociated head and neck cancer. J Immunother Cancer 2015;3:P419.

35. Chen S, Lv M, Fang S, et al. Poly(I:C) enhanced anticervical cancer immunities induced by dendritic cellsderived exosomes. Int J Biol Macromol 2018;113:1182-7.

36. Langers I, Renoux V, Reschner A, et al. Natural killer and dendritic cells collaborate in the immune response induced by the vaccine against uterine cervical cancer. Eur J Immunol 2014;44:3585-95.

37. Xiao M, Feng Y, Cao G, et al. A novel MtHSP70FPR1 fusion protein enhances cytotoxic T lymphocyte responses to cervical cancer cells by activating human monocyte-derived dendritic cells via the p38 MAPK signaling pathway. Biochem Biophys Res Commun 2018;503:2108-16. 
38. Iwata T, Fujii T, Morii K, et al. Cytokine profile in cervical mucosa of Japanese patients with cervical intraepithelial neoplasia. Int J Clin Oncol 2015;20:126-33.

39. Cai D, Yan L, Guo R, et al. Correlation of HPV types with Th17 and Treg cells in cervical cancer. Chinese Journal of Clinical Oncology 2016;43:1099-102.

40. Torres-Poveda K, Burguete-Garcia AI, Bahena-Roman M, et al. Risk allelic load in Th2 and Th3 cytokines genes as biomarker of susceptibility to $\mathrm{HPV}-16$ positive cervical cancer: a case control study. BMC Cancer 2016;16:330.

41. Zhang T, Jiao J, Jiao X, et al. Aberrant frequency of TNFR2(+) Treg and related cytokines in patients with CIN and cervical cancer. Oncotarget 2018;9:5073-83.

42. Zhang J, Chen H, Yang X, et al. Defective TRAF3 modulates alternative NF-kB signaling and cytokine expression to promote cancer cell survival in HPV positive head and neck cancer (TUM10P.1049). J Immunol 2015:194:30.

43. Lam JO, Bream JH, Sugar EA, et al. Association of serum cytokines with oral HPV clearance. Cytokine 2016;83:85-91.

44. Iuliano M, Mangino G, Chiantore MV, et al. Human Papillomavirus E6 and E7 oncoproteins affect the cell microenvironment by classical secretion and extracellular vesicles delivery of inflammatory mediators. Cytokine 2018;106:182-9.

45. Woodham AW, Yan L, Skeate JG, et al. T cell ignorance is bliss: $\mathrm{T}$ cells are not tolerized by Langerhans cells presenting human papillomavirus antigens in the absence of costimulation. Papillomavirus Res 2016;2:21-30.

46. van der Burg SH, de Jong A, Welters MJ, et al. The status of HPV16-specific T-cell reactivity in health and disease as a guide to HPV vaccine development. Virus Res 2002;89:275-84.

47. Da Silva DM, Woodham AW, Skeate JG, et al. Langerhans cells from women with cervical precancerous lesions become functionally responsive against human papillomavirus after activation with stabilized Poly-I:C. Clin Immunol 2015;161:197-208.

48. Leung J, Suh WK. The CD28-B7 Family in Anti-Tumor Immunity: Emerging Concepts in Cancer Immunotherapy. Immune Netw 2014;14:265-76.

49. Lee SJ, Yang A, Wu TC, et al. Immunotherapy for human papillomavirus-associated disease and cervical cancer: review of clinical and translational research. J Gynecol Oncol 2016;27:e51.

50. Chapoval AI, Ni J, Lau JS, et al. B7-H3: a costimulatory molecule for $\mathrm{T}$ cell activation and IFN-gamma production.
Nat Immunol 2001;2:269-74.

51. Chen X, Wang L, Wang W, et al. B7-H4 facilitates proliferation of esophageal squamous cell carcinoma cells through promoting interleukin-6/signal transducer and activator of transcription 3 pathway activation. Cancer Sci 2016;107:944-54.

52. Li Y, Zhang J, Han S, et al. B7-H3 promotes the proliferation, migration and invasiveness of cervical cancer cells and is an indicator of poor prognosis. Oncol Rep 2017;38:1043-50.

53. Stevanovic S, Draper LM, Langhan MM, et al. Complete regression of metastatic cervical cancer after treatment with human papillomavirus-targeted tumor-infiltrating $\mathrm{T}$ cells. J Clin Oncol 2015;33:1543-50.

54. van Poelgeest MI, Visconti VV, Aghai Z, et al. Potential use of lymph node-derived HPV-specific T cells for adoptive cell therapy of cervical cancer. Cancer Immunol Immunother 2016;65:1451-63.

55. Menon S, Shin S, Dy G. Advances in cancer immunotherapy in solid tumors. Cancers 2016;8:106.

56. Hamid O, Robert C, Daud A, et al. Safety and tumor responses with lambrolizumab (anti-PD-1) in melanoma. N Engl J Med 2013;369:134-44.

57. Callahan MK, Wolchok JD. At the bedside: CTLA-4- and PD-1-blocking antibodies in cancer immunotherapy. J Leukoc Biol 2013;94:41-53.

58. Mkrtichyan M, Najjar YG, Raulfs EC, et al. Anti-PD-1 synergizes with cyclophosphamide to induce potent antitumor vaccine effects through novel mechanisms. Eur J Immunol 2011;41:2977-86.

59. Lyford-Pike S, Peng S, Young GD, et al. Evidence for a role of the PD-1:PD-L1 pathway in immune resistance of HPV-associated head and neck squamous cell carcinoma. Cancer Res 2013;73:1733-41.

60. Assal A, Kaner J, Pendurti G, et al. Emerging targets in cancer immunotherapy: beyond CTLA-4 and PD-1. Immunotherapy 2015;7:1169-86.

61. Eskander RN, Tewari KS. Immunotherapy: an evolving paradigm in the treatment of advanced cervical cancer. Clin Ther 2015;37:20-38.

62. Gunn GR, Zubair A, Peters C, et al. Two Listeria monocytogenes vaccine vectors that express different molecular forms of human papilloma virus-16 (HPV16) $\mathrm{E} 7$ induce qualitatively different $T$ cell immunity that correlates with their ability to induce regression of established tumors immortalized by HPV-16. J Immunol 2001;167:6471-9.

63. Kawana K, Adachi K, Kojima S, et al. Oral vaccination 
against HPV E7 for treatment of cervical intraepithelial neoplasia grade 3 (CIN3) elicits E7-specific mucosal immunity in the cervix of CIN3 patients. Vaccine 2014;32:6233-9.

64. Ghamande SA, Platt D, Wheatley D, et al. Phase I study evaluating high-dose treatment with ADXS11-001, a Listeria monocytogenes-listeriolysin O (Lm-LLO) immunotherapy, in women with cervical cancer. J Clin Oncol 2016;34:e14580.

65. Basu P, Mehta AO, Jain MM, et al. ADXS11-001 immunotherapy targeting HPV-E7: Final results from a phase 2 study in Indian women with recurrent cervical cancer. J Clin Oncol 2014;32:5610.

66. Borysiewicz LK, Fiander A, Nimako M, et al. A recombinant vaccinia virus encoding human papillomavirus types 16 and 18, E6 and E7 proteins as immunotherapy for cervical cancer. Lancet 1996;347:1523-7.

67. Kaufmann AM, Stern PL, Rankin EM, et al. Safety and immunogenicity of TA-HPV, a recombinant vaccinia virus expressing modified human papillomavirus (HPV)-16 and HPV-18 E6 and E7 genes, in women with progressive cervical cancer. Clin Cancer Res 2002;8:3676-85.

68. Khan S, Bijker MS, Weterings JJ, et al. Distinct uptake mechanisms but similar intracellular processing of two different toll-like receptor ligand-peptide conjugates in dendritic cells. J Biol Chem 2007;282:21145-59.

69. Kenter GG, Welters MJ, Valentijn AR, et al. Phase I immunotherapeutic trial with long peptides spanning the E6 and E7 sequences of high-risk human papillomavirus 16 in end-stage cervical cancer patients shows low toxicity and robust immunogenicity. Clin Cancer Res 2008;14:169-77.

70. Kenter GG, Welters MJ, Valentijn AR, et al. Vaccination against HPV-16 oncoproteins for vulvar intraepithelial neoplasia. N Engl J Med 2009;361:1838-47.

71. Parmiani G, Russo V, Maccalli C, et al. Peptide-based

Cite this article as: Zhang L, Mao Z, Lai Y, Wan T, Zhang K, Zhou B. A review of the research progress in T-lymphocyte immunity and cervical cancer. Transl Cancer Res 2020;9(3):2026-2036. doi: 10.21037/tcr.2020.01.33 vaccines for cancer therapy. Hum Vaccin Immunother 2014;10:3175-8.

72. de Jong A, O'Neill T, Khan AY, et al. Enhancement of human papillomavirus (HPV) type 16 E6 and E7-specific T-cell immunity in healthy volunteers through vaccination with TA-CIN, an HPV16 L2E7E6 fusion protein vaccine. Vaccine 2002;20:3456-64.

73. Einstein MH, Kadish AS, Burk RD, et al. Heat shock fusion protein-based immunotherapy for treatment of cervical intraepithelial neoplasia III. Gynecol Oncol 2007;106:453-60.

74. Cai L, Zhang J, Zhu R, et al. Protective cellular immunity generated by cross-presenting recombinant overlapping peptide proteins. Oncotarget 2017;8:76516-24.

75. Yang B, Jeang J, Yang A, et al. DNA vaccine for cancer immunotherapy. Hum Vaccin Immunother 2014;10:3153-64.

76. Maldonado L, Teague JE, Morrow MP, et al. Intramuscular therapeutic vaccination targeting HPV16 induces T cell responses that localize in mucosal lesions. Sci Transl Med 2014;6:221ra13.

77. Trimble CL, Morrow MP, Kraynyak KA, et al. Safety, efficacy, and immunogenicity of VGX-3100, a therapeutic synthetic DNA vaccine targeting human papillomavirus 16 and $18 \mathrm{E} 6$ and $\mathrm{E} 7$ proteins for cervical intraepithelial neoplasia 2/3: a randomised, double-blind, placebocontrolled phase $2 \mathrm{~b}$ trial. Lancet 2015;386:2078-88.

78. Thornburg C, Boczkowski D, Gilboa E, et al. Induction of cytotoxic $\mathrm{T}$ lymphocytes with dendritic cells transfected with human papillomavirus E6 and E7 RNA: implications for cervical cancer immunotherapy. J Immunother 2000;23:412-8.

79. Zhu Y, Zheng Y, Mei L, et al. Enhanced immunotherapeutic effect of modified HPV16 E7-pulsed dendritic cell vaccine by an adeno-shRNA-SOCS1 virus. Int J Oncol 2013;43:1151-9. 